The Astrophysical JournaL, 462:813-824, 1996 May 10

(C) 1996. The American Astronomical Society. All rights reserved. Printed in U.S.A.

\title{
RADIO MORPHOLOGIES OF PLANETARY NEBULAE
}

\author{
O. B. AAQUIST ${ }^{1}$ AND SUN KwoK \\ Department of Physics and Astronomy, University of Calgary, Calgary, Alberta, Canada T2N 1N4 \\ Received 1994 November 28; accepted 1995 November 14
}

\begin{abstract}
We have examined the morphologies of planetary nebulae observed in the recent very large array (VLA) surveys. The high angular resolution of the VLA has allowed the extension of morphological classification to a much more compact population than before. The radio images of planetary nebulae are analyzed in terms of the prolate ellipsoidal shell (PES) model. Simulated radio images are produced using various asymmetry parameters in the PES model. Using these models as a guide, the radio images of planetary nebulae are classified into different morphological classes. No significant differences can be found between the statistics of morphological classes of high surface brightness/compact and low surface brightness/extended planetary nebulae, or with those classified by optical means. We conclude that the morphology of planetary nebulae is determined early in their evolution. The degree of asymmetry planetary nebulae inherited from their asymptotic giant branch progenitors is probably as important in determining the asymmetry observed in planetary nebulae as the shaping done by interacting winds during the planetary nebula phase.
\end{abstract}

Subject heading: planetary nebulae: general

\section{INTRODUCTION}

Radio continuum images are well suited to reveal the overall morphology of planetary nebulae (PNs) because the emission at radio wavelengths is due largely to thermal free-free emission. Since free-free emission is only weakly dependent on electron temperature, the observed intensity distribution can be readily interpreted in terms of the ionized gas distribution within the nebula, as long as the nebula is optically thin at the observing frequency. The radio images also have the advantage of being absolutely calibrated, and therefore they give a quantitative representation of the distribution of density (Pottasch 1995). A new morphological scheme, based on the radio images, is desirable because it can lead to a more quantitative study of the morphological distribution of PNs. The statistical distribution of morphological types can be correlated to other physical parameters (e.g., age, mass) to test theories on the origin of different morphologies of PNs.

The advent of radio interferometry offers higher angular resolution than is possible in the visible part of the spectrum. Recent very large array $\left(\mathrm{VLA}^{2}\right)$ surveys of PNs at $\lambda-6$, 3 , and $2 \mathrm{~cm}$ wavelengths have produced radio images of PNs with angular resolutions of 0"1-0"4 (Kwok 1985; Aaquist \& Kwok 1990 [hereafter AK90], 1991; Kwok \& Aaquist 1993). These high-resolution surveys have also identified nebulae of high surface brightness, and therefore candidates of young PNs (Kwok 1983). A similar survey of extended PNs was carried out by Zijlstra, Pottasch, \& Bignell (1989, hereafter ZPB), using the VLA in more compact configurations. As the objects observed by ZPB generally have lower radio surface brightness, they probably represent a sample of more evolved PNs. It would be interesting to compare the morphologies of the PNs observed in these two surveys in order to detect possible change in structure between young and evolved PNs. It is

\footnotetext{
${ }^{1}$ Current address: University transfer division, The College of New Caledonia, Prince George, BC, Canada V2N 1 P8.

${ }^{2}$ The VLA is a facility of the National Radio Astronomy Observatory, which is operated by Associated Universities, Inc. under cooperative agreement with the National Science Foundation.
}

hoped that such an examination will help in our understanding of the early evolution of PNs.

\section{THE PHYSICAL ORIGIN OF ASYMMETRIES OBSERVED IN PLANETARY NEBULAE}

Over the last decade, it has been demonstrated that the shell structures and the nonspherical shapes of PNs are caused by the interactions of a fast central star wind and the remnant of a slow wind from the asymptotic giant branch (AGB) (Kwok, Purton, \& FitzGerald 1978; Balick 1987). If both the fast wind and the slow wind are isotropic, the resultant PN will have spherical symmetry. In that case, the speed of the swept-up shell can be determined from simple hydrodynamic principles through energy, momentum, and mass conservation at the interface of the fast wind and the AGB wind (Volk \& Kwok 1985; Schmidt-Vogt \& Köppen 1987a, b), and the geometrical thickness of the swept-up shell can be determined if the shell's density law and the density jump between the swept-up shell and the undisturbed AGB material are known. If either of the fast wind, slow wind, or radiation field are not isotropic, then the nebula will take on a shape reflecting the nature of the asymmetry. Of the three, the slow wind seems to be the most likely candidate to carry significant asymmetry, although no accepted theory exists which explains the origin of the asymmetry (see Livio 1995). By assuming modest and smooth density variations as a function of polar (latitude) angle $(\theta)$, Kahn \& West (1985) and Icke (1988) demonstrated that the inner boundary of the swept-up shell could range in shape from ellipsoidal to figure " 8 ." Icke (1988) illustrates that for cylindrical symmetry the shock evolves toward a fixed shape in a finite time, after which the shock changes only by expanding with a constant scale factor. If this time is very short (e.g., a few $\times 10^{2} \mathrm{yr}$ ), then the shapes of the young PNs from AK90 should find their counterparts among the more evolved larger PNs. If cylindrical symmetry is not maintained in real nebulae, conspicuous structural differences may become noticeable between these two groups of objects.

The morphology of a nebula depends not only on the shape of the shocked gas, but also on the thickness of the 
ionized region, $\Delta R_{i}=R_{i}-R_{s}$, along the shell. Here $R_{i}$ is the radius of the outer edge of the ionized zone, and $R_{s}$ is the radius of the inner edge of the nebula. This thickness is a strong function of time because it depends heavily on the number of ionizing photons supplied by the central star. If the ionization front enters the unshocked halo, a significant portion of the halo is likely to become ionized. If the density jump at the shell-halo interface is small, then it should be possible to detect the inner part of this halo with the VLA. For a density jump which is much greater than four, the radio halo would be difficult to detect with the limited dynamic range in the "snapshot" mode.

Significant progress has been made recently in the modeling of the dynamics of PNs by including the effects of radiation in the ionization and cooling of the nebulae. Results of these models have been very successful in reproducing the optical morphologies of PNs (Frank et al. 1993).

\section{MORPHOLOGICAL CLASSES IN TERMS OF THE PES MODEL}

If we address the problem of the morphology of PNs from an empirical point of view and examine the radio images of PNs, we find that the many observed morphologies can be explained in a simple empirical geometric model. This model, referred to here as the prolate ellipsoidal shell (PES) model, was first discussed by Masson (1989, 1990). A spherical shell with both radial and latitudedependent density gradients is ionized by a central star to various depths. The resulting ionized shell, when projected onto the plane of the sky and viewed at different angles, will result in a variety of morphologies seen in PNs. A detailed discussion on the PES model and the production of simulated images using different parameters is given in the Appendix.

The usefulness of an empirical model like the PES model is that it can be used to classify the morphology of PNs in a consistent manner and can be used as a model for the testing of detailed dynamical/radiative models of PNs. In the following section, we will show that most of the PNs can be related back to the PES structure. In terms of the threedimensional model, there are basically only three types which can be discerned visually: the spherical shell, the ellipsoidal, and the butterfly. They can be created by varying density ratios of one single basic PES model.

\subsection{Definition of Morphological Classes}

The typical PES model images (Fig. 3) show that the most noticeable differences in appearance are due to line-ofsight angle which gives the images varying degrees of elliptical eccentricity. For all density structures, the images are nearly circular when the inclination angle is less than $15^{\circ}$. The PNs such as K3-62 are classified as circular when no minor axis (clear poles) is visible. When the inclination angle is greater than about $15^{\circ}$ and less than about $60^{\circ}$, the model images show a clear minor axis and central depression, as illustrated in Figure 1 for an inclination of $30^{\circ}$. For these cases, the intensity appears to drop slowly along the major axis and then drop suddenly, giving the impression that the nebula is ionization bounded in that direction; however, this is an illusion created by the line of sight. PNs with these characteristics, such as M1-11, are classified as closed elliptical. When the line-of-sight angle is greater than $60^{\circ}$, the central depression disappears and is replaced by a ridge of flux connecting the two peaks. Along the major axis, the intensity always decreases, giving the impression that the nebula is density bounded along this axis. At high line-of-sight angles, the nebula can look shorter along the major axis because the flux decreases more rapidly from the nebula's center to low emission levels. PNs with these characteristics, such as NGC 6881, are classified as an open elliptical. Open and closed elliptical nebulae are not easily distinguishable if the resolution is poor. For example, $\mathrm{He}$ $2-430$ shows a bridge between the minor axis peaks and is classified as open elliptical; however, the peak centers are less than two beamwidths apart, and any central depression would not be resolved. Therefore, it is possible that our count will be biased toward open elliptical shapes.

Figure 3 shows that when the aximuthal density structure is extreme $(\alpha=4$ and $\beta=0.1)$, the minor axis peaks are very steep and the flux drops rapidly along the major axis. The flux extends always beyond the minor axis quite close to the minor axis peaks which gives the nebula a distinct square, butterfly, or figure " 8 " appearance. PNs with the shape, such as M1-60, are classified as butterfly.

Hence, the PES model gives rise to four recognizable structural classes: circular, elliptical, open elliptical, and butterfly. However, because of low signal-to-noise ratio, poor resolution, and irregularities in the nebulae, the classification is not clear-cut for real PNs. If the signal-to-noise level is low, then the object is simply classified as very noisy; and if the resolution is very poor so that a PN looks like a point or near point source, then the object is simply classified as unresolved.

Most of the real PN images show deviations away from the four basic PES classes. There are three easily recognized asymmetry types consistently seen in PNs: S-type, peak excess, and unbalanced poles. These asymmetries are often recognizable even if the image is poorly resolved or noisy, making them useful classes for PN classifications.

The $S$-type morphology is found in nebulae showing a point symmetry, but the minor axis (joining the peak emission points) is not perpendicular to the major axis as defined by the extended emission, giving the nebula an overall S-like structure. In the radio images of AK90, this type of symmetry can be seen in He 2-477. The effect may be quite subtle, such as in $\mathrm{Hb} \mathrm{4}$, where the $\mathrm{S}$-shape is not clear but the minor and major axis are clearly not perpendicular. The effect is more prominent in the optical images, where the low-level emission is revealed; consequently, the optical images have many more objects with asymmetries of this type (Stanghellini, Corradi, \& Schwarz 1993).

Peak excess occurs when one minor axis peak is significantly brighter than the other. This effect is seen in varying degrees from very prominent, as in K3-13, to very subtle, as in M1-11. When the major axis of a PES with peak excess is directed toward the observer, the nebula takes on the appearance of a blister such as in $\mathrm{Bl} 2-1$. If the asymmetry occurs along the major axis, we refer to it as unbalanced poles. This effect, as seen in M1-25, is often more subtle than peak excess. If no significant asymmetry is discernable, then the image is classified as symmetric, as in M4-18, which is classified as symmetric closed elliptical; the irregularities in this image may be due to the low signal-to-noise ratio. A few objects show more than one type of asymmetry, so the total of the asymmetry subgroups will not add exactly to the total number of PES structures.

In addition to the asymmetry types, a few radio images show signs of halo, such as He 2-440, and ansae, such as 


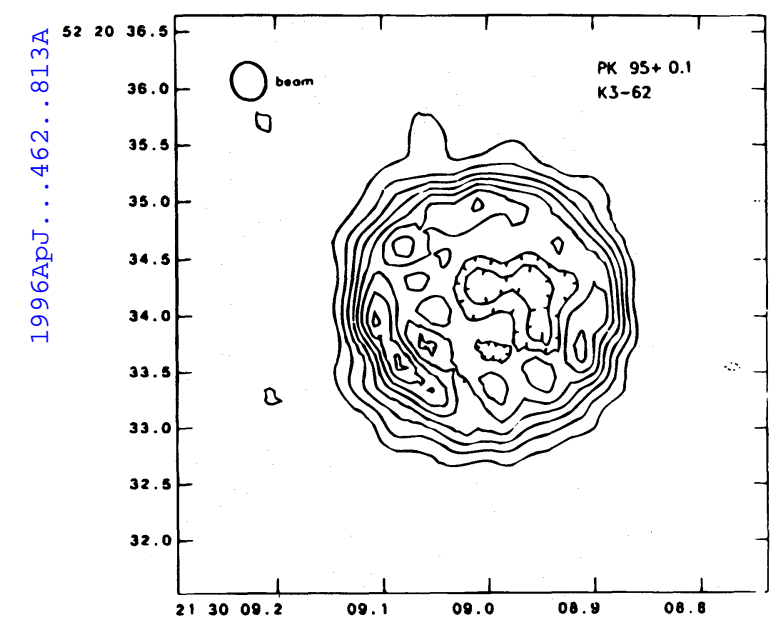

Circular

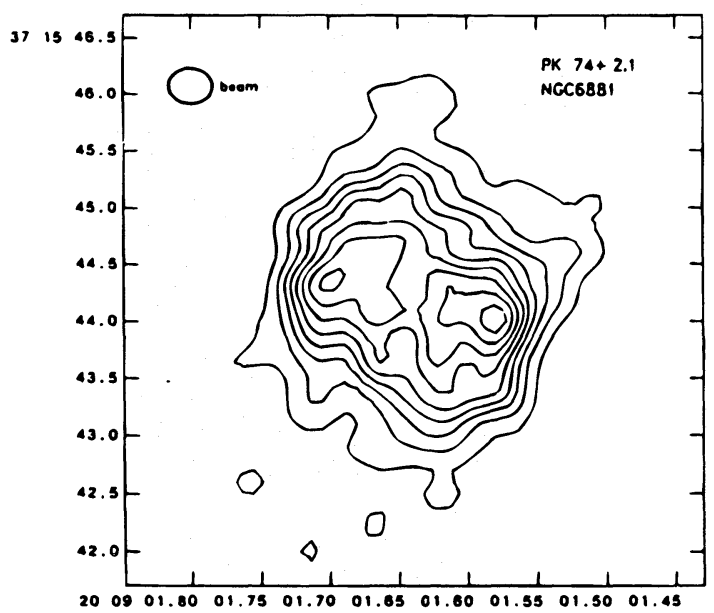

Open Elliptical

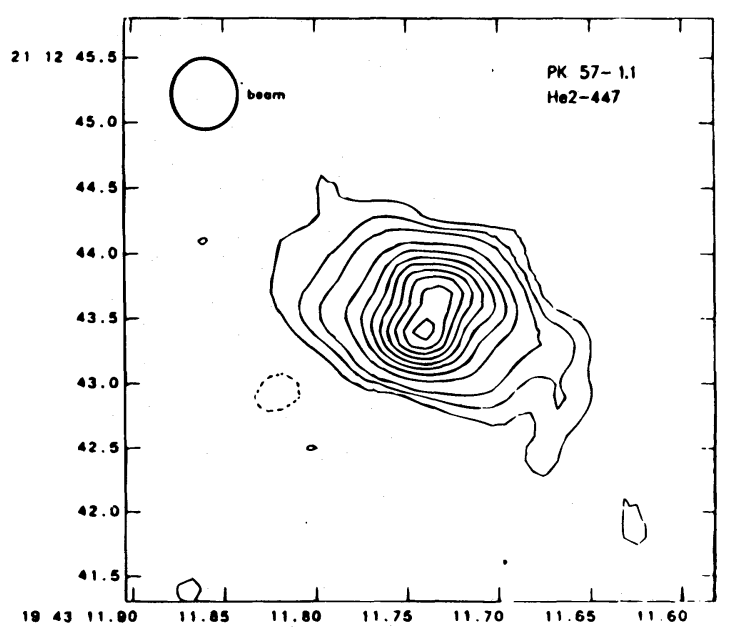

S-type

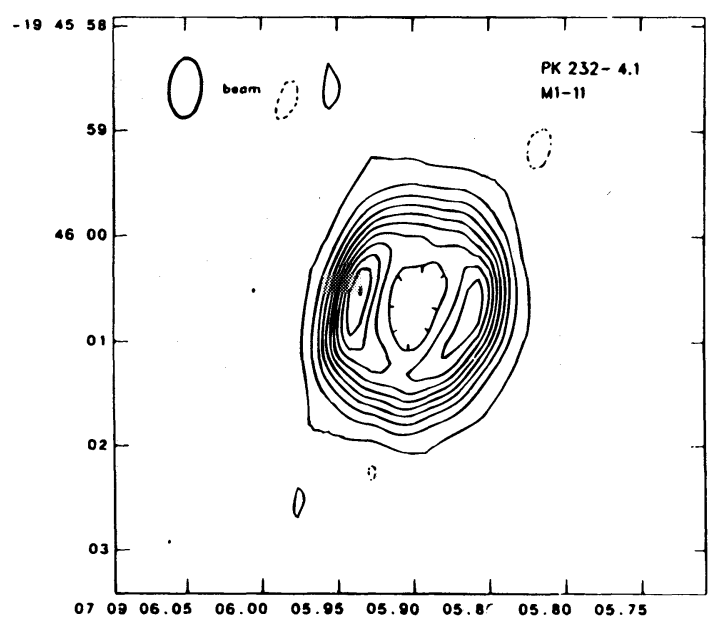

Elliptical

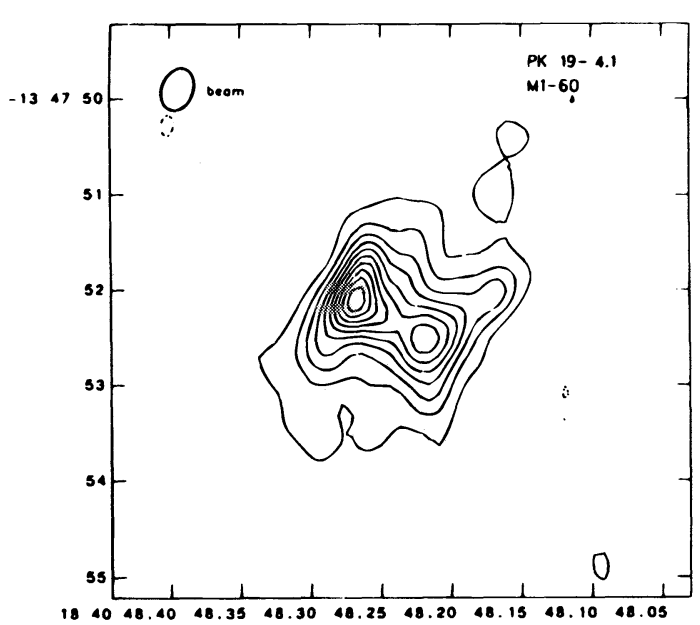

Butterfly

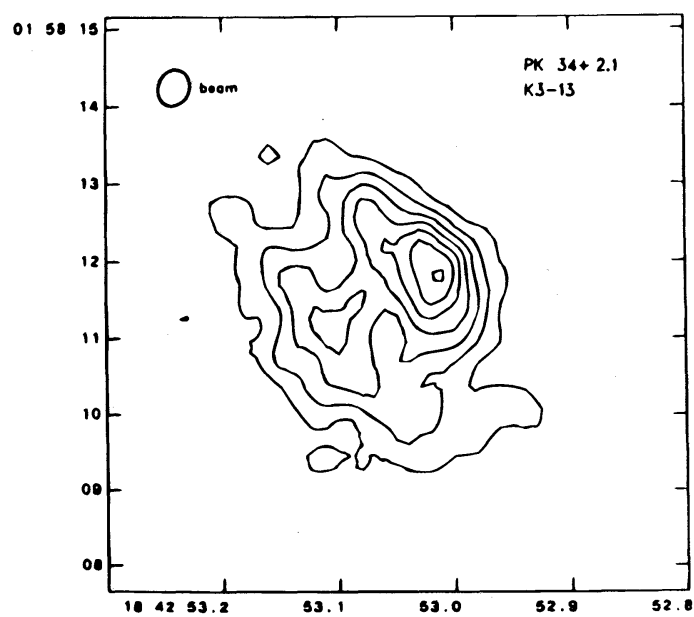

Peak Excess

FIG. 1.-Examples of PNs observed in AK90 illustrating each of the PES morphological class 


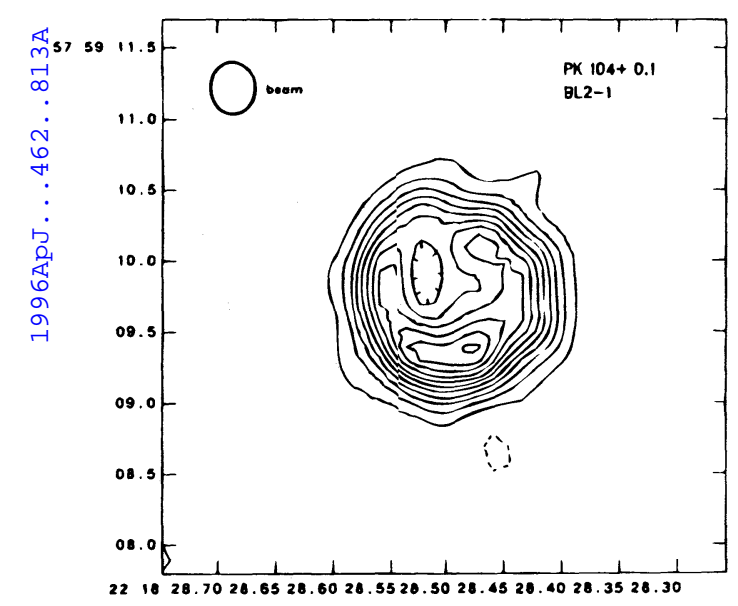

Blister

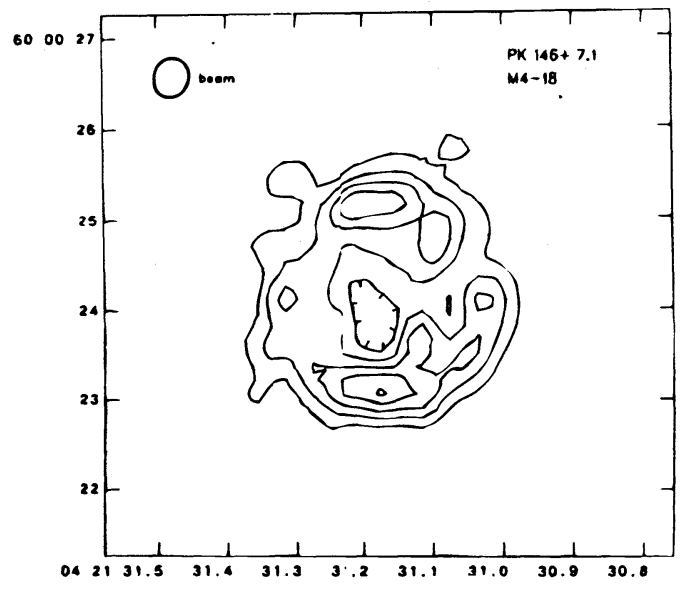

Symmetric

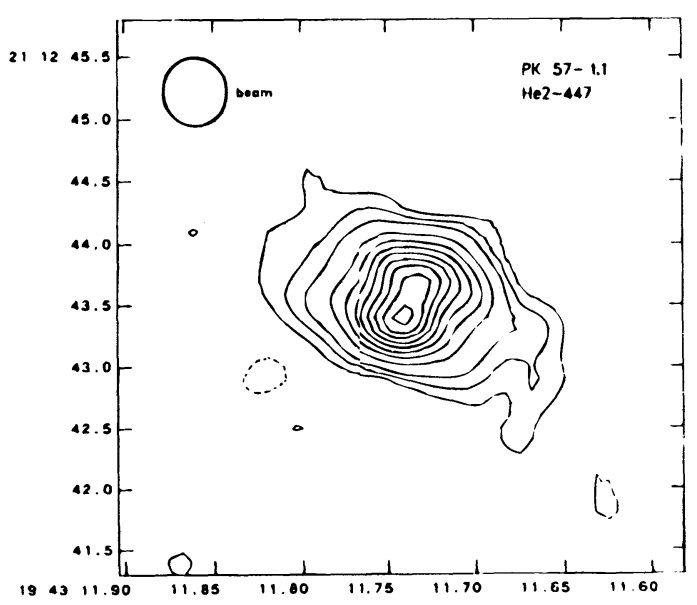

Ansae

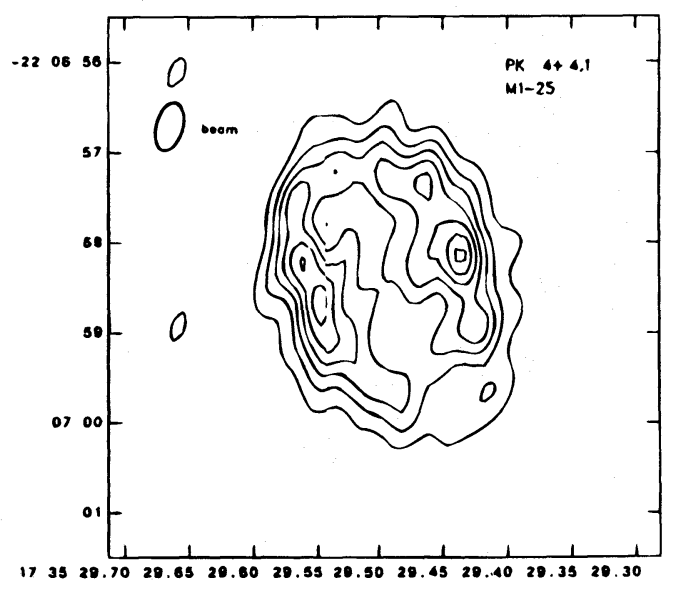

Unbalanced Poles

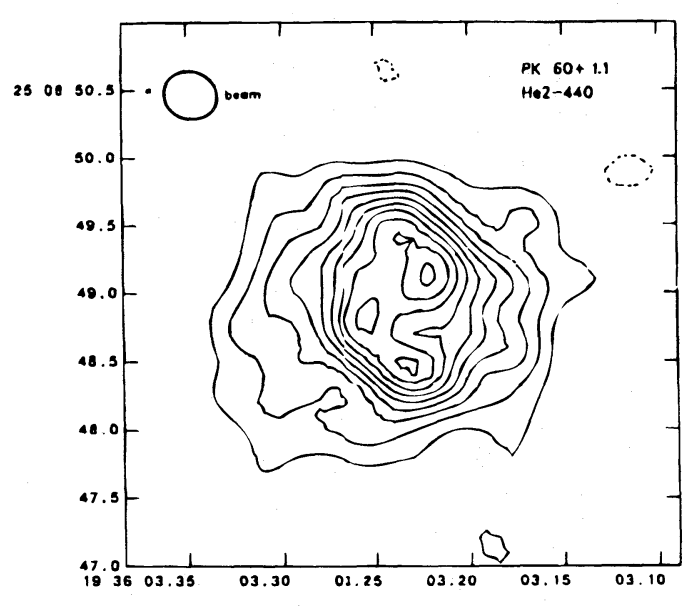

Halo

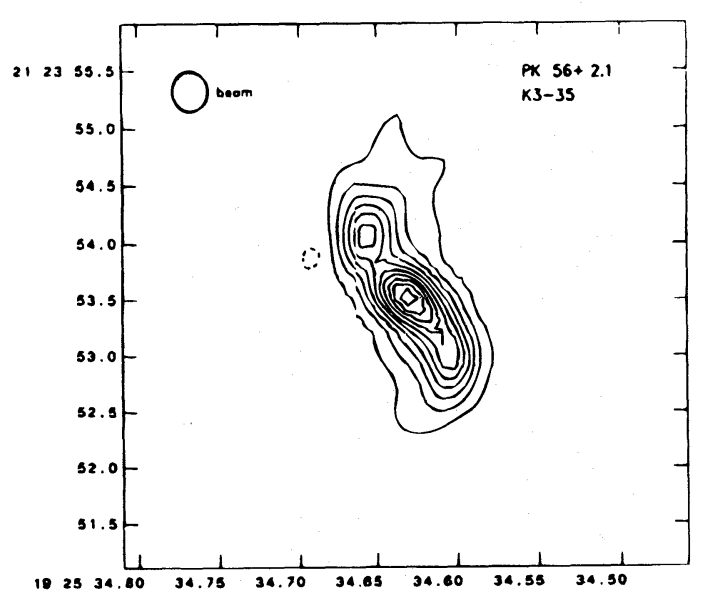

Non-PES

FIG. 1-Continued 
TABLE 1

The Distribution of Morphological Groups BETWEEN DIFFERENT PN SURVEYS

\begin{tabular}{|c|c|c|c|}
\hline Group & AK & ZPB & SCM \\
\hline \multicolumn{4}{|c|}{ Main Structure } \\
\hline PES $\ldots \ldots \ldots \ldots \ldots \ldots \ldots$ & 52 & 42 & 97 \\
\hline Poorly resolved ...... & 10 & 29 & 15 \\
\hline Noisy ............. & 3 & 9 & 1 \\
\hline Unresolved ....... & 25 & 35 & 91 \\
\hline Very Noisy ............ & 19 & 7 & 44 \\
\hline Non-PES $\ldots \ldots \ldots \ldots$ & 4 & 6 & 7 \\
\hline Total .......... & 113 & 128 & 255 \\
\hline \multicolumn{4}{|c|}{ PES Class } \\
\hline Circular ......... & $17 \%$ & $19 \%$ & $25 \%$ \\
\hline Elliptical ........ & $38 \%$ & $36 \%$ & $39 \%$ \\
\hline Open elliptical..... & $37 \%$ & $36 \%$ & $19 \%$ \\
\hline Butterfiy .......... & $8 \%$ & $7 \%$ & $19 \%$ \\
\hline Total........... & 52 & 42 & 97 \\
\hline \multicolumn{4}{|c|}{ Asymmetry Type ${ }^{a}$} \\
\hline S-type symmetry ....... & $8 \%$ & $8 \%$ & $23 \%$ \\
\hline Peak excess .............. & $32 \%$ & $31 \%$ & $19 \%$ \\
\hline Unbalanced poles ...... & $42 \%$ & $40 \%$ & $31 \%$ \\
\hline Symmetric ............. & $26 \%$ & $24 \%$ & $32 \%$ \\
\hline Halos ...................... & $9 \%$ & $8 \%$ & $23 \%$ \\
\hline Total $^{\mathrm{b}} \ldots \ldots \ldots \ldots \ldots$ & 65 & 90 & 111 \\
\hline
\end{tabular}

a Some nebulae are assigned more than one asymmetry types (e.g., halo), resulting in a total of greater than $100 \%$.

bome of the poorly resolved objects are assigned an asymmetry type, resulting in a total larger than the total in the PES class.

$\mathrm{He}-447$. Since these features are generally found at low flux levels, they are not often seen in the radio images.

A few images which do not seem to fit the PES classification, such as K3-35 (Aaquist 1993), are classified as non$P E S$. They are not classified further into symmetric or asymmetric subgroups, nor is any visible halo counted.

\subsection{Statistics of the Morphological Classes}

A summary of the examples of the above morphological groups is shown in Figure 1. The radio images of AK90 and ZPB were classified according to the above scheme. For the nebulae that show the PES form, they are assigned one of the classes of circular, closed elliptical, open elliptical, or butterfly. All these objects, including those that are poorly resolved, are also assessed an asymmetry types: $S$-type, peak excess, unbalanced poles, or symmetric. In addition, some are also assigned as having a halo. The percentage breakdown is shown in Table 1. As can be seen from this table, the morphology statistics of the AK90 and ZPB radio images are practically identical. There is no discernible morphological difference between the two samples.

Note that if the transverse asymmetries (peak excess or unbalanced poles) are caused by interaction with the interstellar medium or some other random process, then there should be no preference as to the direction of the asymmetry through the nebula. Tranverse asymmetries directly toward/away from us will, of course, go unnoticed. However, the radio images seem to favor peak excess over unbalanced poles asymmetry. This probably arises because slight differences in the material distributions within the denser (brighter) material is more noticeable.
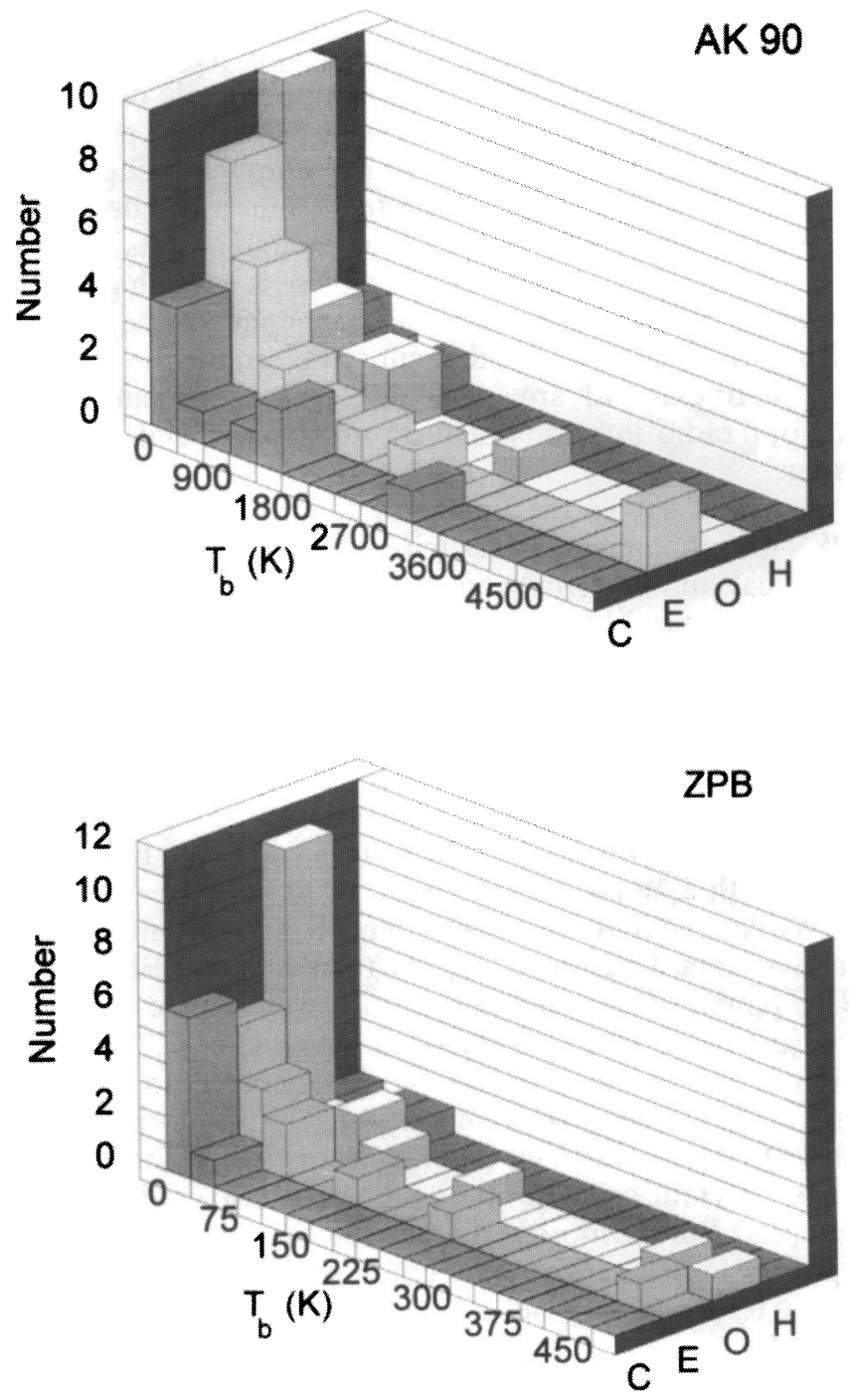

FIG. 2.-Histograms showing the distribution of PNs of different PES classes (C: circular; $\mathrm{E}$ : closed elliptical; $\mathrm{O}$ : open elliptical; and $\mathrm{H}$ : butterfly) with $5 \mathrm{GHz}$ brightness temperature for the VLA survey samples of AK90 (top) and ZPB (bottom). In the AK90 diagram, the last bin of " $\mathrm{E}$ " contains two very high surface brightness objects: NGC $6790\left(T_{b} \sim 6300\right.$ $\mathrm{K})$ and IC $5117\left(T_{b} \sim 7600 \mathrm{~K}\right)$.

On a purely statistical basis, it is not difficult to show that $9.4 \%$ of randomly oriented ellipsoids will have an inclination angle of less than $25^{\circ}$. If their major-to-minor axis ratio is of the order of $1: 2$, this makes them nearly circular in appearance. Of the 52 PES images in AK90, nine objects $(17 \%)$ have circular symmetry. Most of these can be modeled by a nearly spherical shell rather than an end-on ellipsoid. For example, M1-66 cannot be modeled by a thin ellipsoid shell; a thick, nearly spherical shell produces a better visual comparison. There is only one "perfectly" circular image (K3-62) in the VLA surveys. Modeling suggests that it is a spherical shell rather than an end-on ellipsoid. It is interesting to note that if we remove the nebulae that can be modeled by a nearly spherical shell rather than an end-on ellipsoid, then we end up with about the correct percentage of "circular" objects. The existence of purely spherical shell PNs suggests that there is a population of AGB stars with almost perfect spherically symmetric massloss histories.

\section{(C) American Astronomical Society - Provided by the NASA Astrophysics Data System}


It would be interesting to see whether the appropriate distribution of observed minor-to-major axis $(b / a)$ ratios can be explained by a random projection angle of the PES model. For example, this can be achieved by plotting a distribution of the axis ratio of the elliptical nebulae and compared with what is expected from a single model. However, in practice, there are not enough objects for more than two or three bins. Moreover, there is enough apparent variety among the nebulae (asymmetries, extended structures) that it will be difficult to determine the length of the major axis with any confidence. This is particularly true for our radio images, which do not have a high dynamic range.

Figure 2 shows the frequency distributions of the four PES morphological classes (circular, closed elliptical, open elliptical, and butterfly) versus the $5 \mathrm{GHz}$ radio surface temperature. The size of the bins is arbitrary, but it was chosen so that the maximum bin height is about 10 . The bin size for the AK90 and ZPB samples is 300 and 75 , respectively; therefore, almost the entire ZPB sample, can be fitted into the first two bins of the AK90 diagram. Note that the last bin of the AK90 diagram contains all values which exceed $5100 \mathrm{~K}$. The two members which occupy this bin are IC 5117 , with $T_{b}=6627 \mathrm{~K}$, and NGC 6790, with $T_{b}=7125 \mathrm{~K}$.

We can see that the AK90 sample has overall many more high surface brightness objects than in the ZPB sample. This remains true when the entire survey sample is plotted, not just those with PES classifications (see Fig. 3 of AK90). Within each sample, there is no clear correlation between surface brightness (i.e., dynamical age) and morphological class. In other words, we find no clear evidence for morphological evolution with time in these two radio survey samples. Furthermore, evidence for asymmetric morphologies $(\mathrm{E}, \mathrm{O}, \mathrm{H})$ is already present in very high surface brightness (i.e., very young) nebulae.

\section{IONIZATION OR DENSITY BOUNDED?}

The question of whether most PNs are ionization or density bounded has been seriously debated in the literature. Pottasch (1992) suggests that most PNs are ionization bounded, but the detection of halos in many PNs indicate otherwise. From the radio images, the total extent of the ionization along the major axis is difficult to determine. In some cases, the major axis density must be made very low in order for the model to reproduce the shape. These nebulae are likely to be density bounded in this direction. On the other hand, the minor axis profiles most often show the shell to be unresolved, and, generally, there is no evidence of extended emission. This indicates that many of the nebulae are ionization bounded along the minor axis, although a low-level ionized halo cannot be ruled out. Hence, the dimensions of PNs at radio wavelengths are often not well defined along the major axis, since low-level emission is likely to exist in that direction. The diameter of the minor axis seems well defined in most cases. If the shell could be resolved for a substantial number of PNs, then the relative shell thickness (across the minor axis) would serve as a useful distance-independent parameter. This is particularly true since the apparent minor axis diameter and shell thickness will not be greatly influenced by the orientation of the nebula. For the radio images in AK90, however, the shell is either unresolved, or the signal-to-noise ratio is low. Hence, the images are generally not suitable for examining the density structure within the ionized shell in more detail than described in this paper. Higher frequency observations with greater $u-v$ coverage are required for the unresolved shells. With better images, and careful modeling, it may be possible to deduce the density structure of many PNs which fit the PES morphological scheme.

The steep isophotes, especially along the minor axis, suggest an ionization-bounded condition in that direction. However, such steep gradients can also be the result of a shock boundary, and the existence of low-level emission beyond the outer edge cannot be ruled out because the radio data is sensitivity limited. In fact, optical data often show extended emission beyond the steep radio isophotes, and many PNs have been found through the CCD imaging to have extended halos (Chu, Jacoby, \& Arendt 1987). While halos have generally not been detected around the compact PNs in the AK90 sample, they could probably be seen given enough angular resolution (e.g., by the Hubble Space Telescope). A few objects do show extended halos in their radio images, indicating that the ionization front has entered the unshocked AGB wind.

\section{COMPARISONS WITH OPTICAL IMAGES}

An analysis by Evans \& Thackeray (1950) of resolved optical images of PNs showed that $45 \%$ have biaxial symmetry, $10 \%$ have circular symmetry, and $30 \%$ have irregular symmetry; Z-type (equivalent to our S-type) symmetry is seen in $10 \%$ of the nebulae, and helical symmetry is seen in $5 \%$. These statistics agree with the above classification statistics for the radio images. Because S-type and helical symmetries are difficult to see in the radio images, they would tend to be sorted with the PES group. However, slight S-type symmetry can be seen in three $(\sim 4 \%)$ of the AK90 images: Hb 4, He 2-447, and IC 4634. The helical symmetry is not seen in the radio images; if this symmetry is present, then they would have been grouped with the peak excess class.

Khromov \& Kohoutek (1967) claim that of 295 wellresolved images in the PK catalog, $263(89 \%)$ can be classified in terms of an open-ended cylinder viewed at different angles (a more simplistic version of the PES model). A similar high percentage is found by us for the radio data using the PES model (see Table 1). Khromov \& Kohoutek attribute the apparent statistical overabundance of ringshaped PNs to be a selection effect. Supposedly, this shape is more noticeable in photographs, making the likelihood of discovery greater. If this is so, then the compact $\mathrm{PN}$ radio survey of AK90 should have no such bias because the majority of the objects therein were classified on the basis of emission-line spectra rather than by their photographic appearance. As discussed in $\S 3.2$, the overabundance of the circular objects is in fact real.

Greig (1971) studied 160 PNs from various sources and correlated their morphology to other characteristics of the objects, such as type of central star and nebular excitation. He concluded that there are principally only two major groups of PNs, class B and class $C$. Class $B$ has large ansae, tubular, or filamentary structures. Many members that are bipolar are brightest at the ends of, or along, the minor axis with sharp cutoff in brightness beyond the minor axis. Furthermore, this type often has strong forbidden lines of [N II], [O II], and [O I] relative to the Balmer lines; many of the nuclei are of type WC (implying more advanced nuclear processing), and they seem to be located at a significantly lower mean galactic latitude than the other type. 
Class $C$ PNs have a centric increase in brightness, and they are more smooth in appearance than class $B$. They have weaker forbidden line intensities, they occur at significantly higher mean galactic latitudes, and most often they have WN- or Of-type nuclei. Therefore, the possibility of inferring other characteristics from the shape alone may be possible. Unfortunately, very few of the compact PNs in AK90 have excitation classifications: 10 are classified as class $B$, and 12 are classified as class $C$. This makes statistical comparison difficult. Moreover, the radio images are not suitable for detecting filamentary structures because of the small number of resolving elements.

Westerlund \& Henize (1967) carried out an examination of 151 optical images in the light of $\mathrm{H} \alpha$ which indicated that young objects (small and bright) showed greater structural regularity. They suggest that this could represent either a resolution effect or an evolution effect. From the above discussions, the former case seems to apply.

Balick (1987) using CCD images of 51 PNs taken in the light of low-, moderate-, and high-ionization emission lines, organized the nebulae into an evolutionary sequence consistent with the idea of shaping of PNs by interacting winds. If this approach is viable, then AK90, which observed mainly compact and young nebulae, should contain very few or none of the morphologies which Balick considers to be evolved objects: the butterfly-shaped nebulae. Taking Balick's images to be representative of the larger images, one finds that approximately the same percentage of this type of nebulae is found in both the AK90 survey images and these optical images. This indicates that the butterfly nebulae are not purely an evolutionary phenomenon (through which all PNs will pass). Furthermore, there are many examples in which the $\mathrm{H} \alpha$ images of the large PNs are very similar in appearance to the radio images of compact PNs. This is again supportive of the idea that the shapes of PNs develop very early in their evolution, after which they change only in relative size as they age.

Two hundred fifty-five narrow-band $\mathrm{H} \alpha$ and [O III] images of PNs have been obtained by Schwarz, Corradi, \& Melnick (1992, hereafter SCM). Owing to the higher dynamic range of the CCD images, faint halos are often seen. The angular sizes of the nebulae image ranged from $\sim 1^{\prime \prime}$ to $4^{\prime}$. These images were classified by Stanghellini et al. (1993) into the following classes: (i) stellar, (ii) elliptical, (iii) bipolar, (iv) point-symmetry, and (v) irregular. They define bipolar as nebulae that show a "waist," which we call butterfly. Their elliptical and bipolar classes together correspond to our PES class, and point-symmetric is equivalent to our $S$-type.

We have attempted to classify the SCM images according to our classification scheme. The results are given in Table 1. In comparison with the radio samples of $A K 90$ and $\mathrm{ZPB}$, we note the following:

1. There are more circular PNs in the optical images;

2. There are more butterfly PNs in the optical images;

3. There is an abundance of open elliptical in the radio images;

4. There are more symmetric and fewer peak excess and unbalanced poles in the optical, with the disparity being much greater in the former case;

5. There are many more optical PNs with S-type asymmetries;

6. There are considerably more halos in the optical images.

Most of these differences can be accounted for in terms of dynamic range, sensitivity, and image presentation. The above list of differences can be explained as follows:

1. There are seven circular optical PNs which are very weak. Such weak sources would go undetected in the radio region. When these seven objects are removed from the data set (counted as very noisy objects), the percentage of circular PNs decreases to $19 \%$ which is in line with what is seen in the radio images.

2. Butterfly nebulae are easier to identify in the optical because detection of the faint extended emission requires a high sensitivity. Hence, some of the butterfly PNs in the radio might be misclassified as open elliptical. For example, six open elliptical PNs in the AK90 sample which have very short major axes could be butterfly PNs. This would have increased the percentage of butterfly PNs to $19 \%$ and reduced the open elliptical to $25 \%$, which is more in line with the optical images.

3. Point (2) above addresses the excess of open elliptical seen in the radio images. There will also tend to be an abundance of open elliptical in the radio images due to the poor resolution in the radio. Specifically, poor resolution obscures a central depression between the two minor axis peaks, and if no central depression is seen, the object usually is classified as an open elliptical.

4. The existence of more symmetric optical images can be explained by poor dynamic range in the published SCM images. Often the optical gray-scale images are saturated, which tends to obscure asymmetries. This saturation will tend to obscure the peak excess more than the unbalanced poles because the flux is usually greater along the minor axis (and hence will saturate first).

5. There are many more optical PNs with S-type symmetry because this type of symmetry is most often seen in the low-level emission. Since the optical detector has a greater sensitivity than the radio detectors, it follows that there should also be more S-type objects seen in the optical.

6. The argument here is similar to that in (5) above.

Given the systematic differences between the optical and radio techniques, and the subjective nature of the classification process itself, it cannot be concluded that there are any statistical morphological differences within the various categories among the three surveys.

\section{WHEN DOES THE SHAPING OF PNS BEGIN?}

In the scenario of PN morphological evolution as outlined by Balick (1987), PNs evolve from slight to extreme bipolar shapes as the result of interacting winds. A "butterfly" PN is expected to be at a later stage of PN evolution than an elliptical one. Such an evolutionary sequence is not obvious in our analysis of the radio survey images ( $\$ 3.2$ and 5). Some compact and high surface brightness nebulae (e.g., $\mathrm{Hu}$ 2-1) have extreme asymmetries, although the dynamical age of $\mathrm{Hu} 2-1$ is estimated to be only $200 \mathrm{yr}$ (Kwok \& Aaquist 1993). This suggests that another parameter other than age, probably central star mass, is responsible for determining the morphology of the nebula. If an AGB star of higher core mass is losing mass preferentially in the equatorial directions (e.g., as a result of higher rotation rate), then such asymmetric envelope would be evident in very young PNs. Since a strong central star 
wind is not expected to commence until the central star begins emitting a significant amount of $u v$ photons, the morphology of very young nebulae is likely to be shaped by the passing of the ionization front (Frank \& Mellema 1994).

Pottasch (1992) has noted that the morphologies of PNs have not changed noticeably since the work of Curtis (1918). This places some limits on the effects of dynamics/ ionization on the shaping of PNs over a timescale of $80 \mathrm{yr}$. The monitoring of morphological changes in PNs is useful in view of the prediction by the models of Mellema (1995) that significant morphological changes are expected over a timescale of several hundred years.

The morphology of a PN is likely to be the result of the combination of two factors: how much asymmetry was in the remnant AGB envelope (probably controlled by core mass), and how much shaping the nebula has undergone through the process of interacting winds (time evolution). Since PNs of high surface brightness can only come from high core-mass population (which has a short transition time from AGB to PN; see Zhang \& Kwok 1993) and the AK90 sample has a larger fraction of high surface brightness objects (Fig. 2), it is possible that the asymmetries observed in the AK90 sample are more core mass related. On the other hand, if the ZPB sample has lower core masses on the average, then the asymmetries observed could be the result of evolution. Unfortunately, there is no easy way to separate these two factors.

Figure 2 shows that there is no clear correlation between the qualitative PES classes and age of the nebulae. It would be useful to design a scheme that can quantify the degree of ellipticity or asymmetry and to compare this index for the same nebula observed at different epochs. However, the construction of such an index which is independent of resolution is difficult. For the brighter, well-resolved objects, qualitative changes in the morphology may be detectable, since some of the AK90 objects were observed almost $10 \mathrm{yr}$ ago. Assuming an expansion speed of $25 \mathrm{~km}$ $\mathrm{s}^{-1}$ and a distance of about $1 \mathrm{kpc}$, there should be an angular expansion of about 5 mas per year. After $10 \mathrm{yr}$, this amounts to $0^{\prime \prime} .05$, which should be measurable with the VLA. A repeat of the radio survey in a few years should yield interesting new information.

\section{SUMMARY AND CONCLUSIONS}

Recent theoretical models of the dynamics of PNs have shown that the interacting winds process can lead to a prolate ellipsoidal shape for the swept-up shell. In this paper, we have shown that many of the well-resolved, higher signal-to-noise ratio radio images of PNs can be satifactorily reproduced in the PES model. However, a variety of azimuthal density gradients had to be used in order to effectively duplicate many of the gross features of individual objects. In other words, a thin, uniformly dense shell is not adequate to explain the morphology of PNs.

Manipulation of the model parameters showed that the central surface brightness of most nearly circular nebulae are too high to be consistent with an end-on ellipsoidal shell structure. Hence, the overabundance of nearly circular objects is not a selection effect. There is a population of PNs which have real spherical shapes, suggesting that certain AGB stars (those of lower core mass?) have spherical mass loss on the AGB.

The radio images suggest that some nebulae could be ionization bounded along the minor axis but density bounded along the major axis. This greatly complicates the debate on whether PNs are ionization bounded or not because one-dimensional models are likely to be inadequate.

Finally, it was illustrated that over the model parameters there appears to be no obvious difference in the distribution of morphological types between the compact PN survey of AK90 and angularly larger images of ZPB. There is also no evidence that the more asymmetric PNs come from an older, more evolved population. Since the AK90 sample contains primarily young PNs, the above observation favors the suggestion that the morphologies of PNs are determined at an early age. This, in turn, implies that the morphologies of PNs are mostly inherited from the AGB stage, or the fast wind must be very efficient in shaping the nebulae soon after ionization begins. The question of whether "nature" or "nurture" is more important in the shaping of PNs can best be answered by comparing the morphologies of proto-PNs and the young PNs discussed here.

We thank an anonymous referee who made several suggestions that led to an improved manuscript. This work is supported by a grant to $\mathrm{S}$. K. from the Natural Sciences and Engineering Research Council of Canada.

\section{APPENDIX}

\section{PARAMETERIZATION OF THE PROLATE ELLIPSOIDAL SHELL MODEL}

The general geometry of the PES model is as follows. A single (hot) star is located interior to a cloud hydrogen gas. The inner region of the nebula is assumed to be empty, supposedly swept clean by a fast wind from the central star (the "hot bubble"). The radius of the inner edge of the gas is given by $R_{1}$; it is a function of the two polar angles $(\theta, \phi)$. The UV photons from the central star ionize the surrounding gas out to the radius $R_{i}$, which depends upon $R_{1}$ and the density structure of the surrounding nebula.

If the shape and size of the inner surface of the swept-up shell is given, then the outer ionized boundary can be calculated by an analysis similar to that carried out for a static Strömgren sphere. The depth of the ionization front along any radial vector is given approximately by the equation

$$
\frac{d L}{d \Omega}=\alpha^{(2)} \int_{R_{s}}^{R_{i}} n(\theta, \phi, r)^{2} r^{2} d r
$$


$i$ $\alpha=1, \beta=0.5$
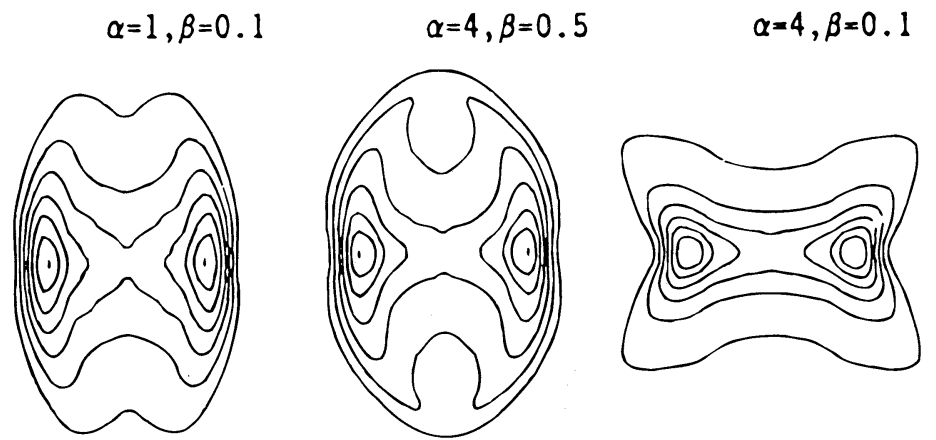

60
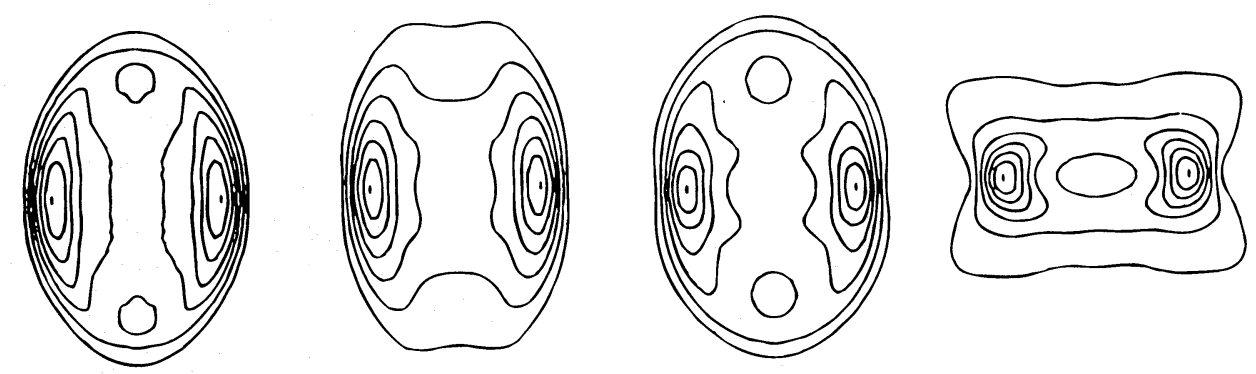

30
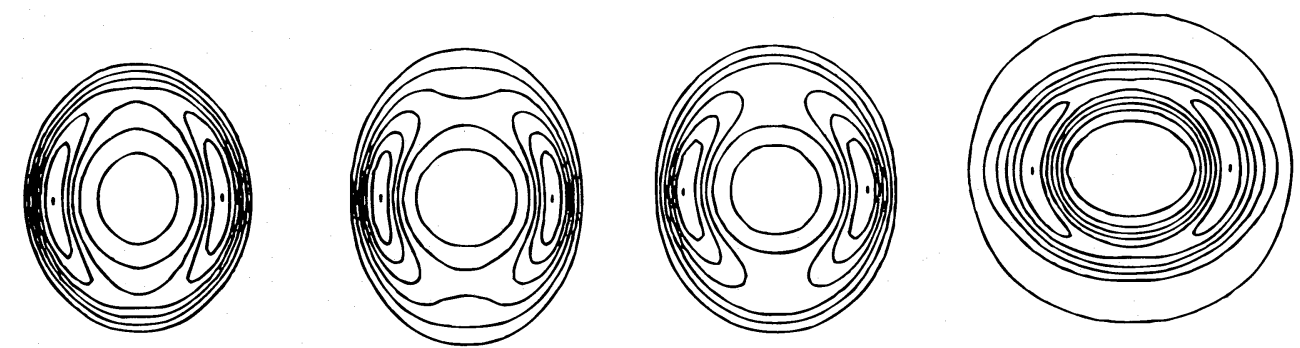

FIG. 3.-Twelve model images for various values of the parameters of the PES model

which equates the number of local ionization photons $(L)$ per unit solid angle $(\Omega), d L / d \Omega$, to the total number of recombinations within the local ionized region. In this equation, $\alpha^{(2)}$ is the recombination coefficient; $n(\phi, \theta, r)$ is the density law; and $\phi, \theta$, and $r$ are the spherical coordinates in the usual sense. The angle $\theta$ is the "latitude" measured from the major axis, and $\phi$ is the "longitude" measured perpendicular to the major axis and the line of sight. $R_{1}$ and $R_{i}$ are both functions of $\phi$ and $\theta$. Little is known about the density law which exists in the swept-up shell. For simplicity, we assume that it is separable, so that $n(\phi, \theta, r)=n_{0} \eta_{\phi} \eta_{\theta} \eta_{r}$; the $\eta$ values are dimensionless quantities describing the density relative to $n_{0}$, which is the density at $(\phi, \theta, r)=\left(0,0, R_{s}\right)$. The choice of the functions $\eta$ will depend upon the density structure of the AGB envelope as well as the interaction of the fast wind with it. Simple radial density laws, such as $\eta_{r}=\left(r / R_{s}\right)^{-\gamma}$, can be integrated to give a simple analytical solution to equation (A1), namely,

$$
\frac{d L}{d \Omega}=\alpha^{2} n_{0}\left(\eta_{\theta}, \eta_{\phi}\right)^{2} R_{1}^{3} \frac{\left(R_{i} / R_{1}\right)^{-2 \gamma+3}-1}{-2 \gamma+3} .
$$

The shape of the inner surface, described by $R_{1}$, may not be a simple ellipsoid, although for certain assumed density distributions in the AGB envelope, ellipsoidal-like inner surfaces theoretically occur (Kahn \& West 1985; Icke 1988; Frank \& Mellema 1994). Once the functions which describe the inner boundary, the radial density law $(\gamma)$, and the minor axis shell thickness $\left(\Delta R_{i}\right)$ are chosen, then the outer ionized boundary, $R_{i}(\phi, \theta)$, follows from equation (A2).

To generate the projected appearance of this model, a prolate ellipsoidal surface was defined within a three-dimensional grid. The cell size relative to the ellipsoid corresponded approximately to that used in the processing of the VLA images in AK90. Equation (A2), with $\gamma=0$, was used to determine if each cell was interior to the ionized region. If it was, then the cell was given a weight between 0 and 1 according to the square of the (somewhat arbitrary) density function

$$
\begin{array}{ll}
\eta_{\theta}=(1-\beta)\left[\left(\frac{2 \theta}{\pi}\right)^{\alpha}+\frac{\beta}{1-\beta}\right] & 0 \leq \theta \frac{\pi}{2}, \\
\eta_{\theta}=(1-\beta)\left[\left(\frac{2 \pi-2 \theta}{\pi}\right)^{\alpha}+\frac{\beta}{1-\beta}\right] & \frac{\pi}{2} \leq \theta \leq \pi .
\end{array}
$$

In this expression, $\alpha$ and $\beta$ are positive constants used to scale the density function: $\alpha$ controls the steepness of the angular density gradient, and $\beta$ is the ratio of the major-to-minor axis density. The weights were summed through the nebula along a 

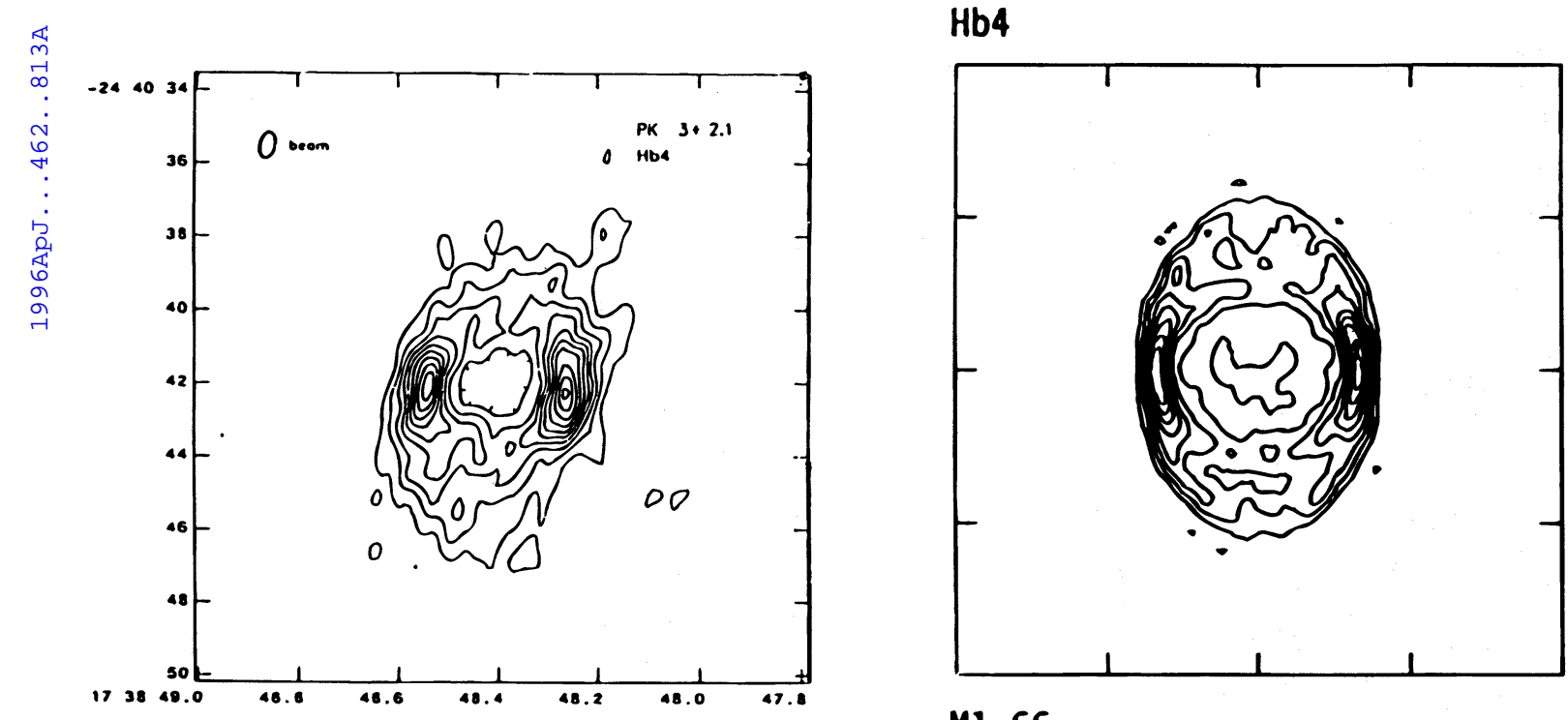

\section{M1-66}
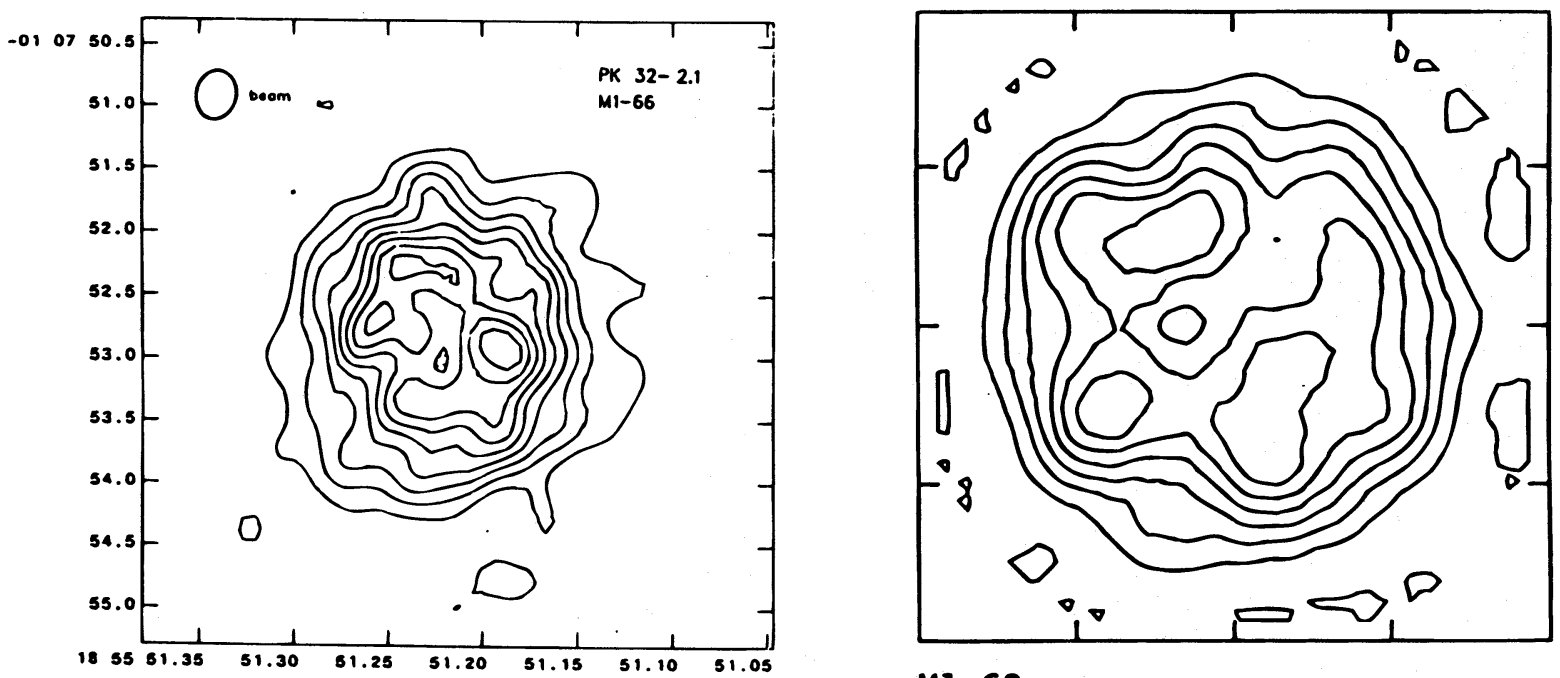

\section{Ml -60}
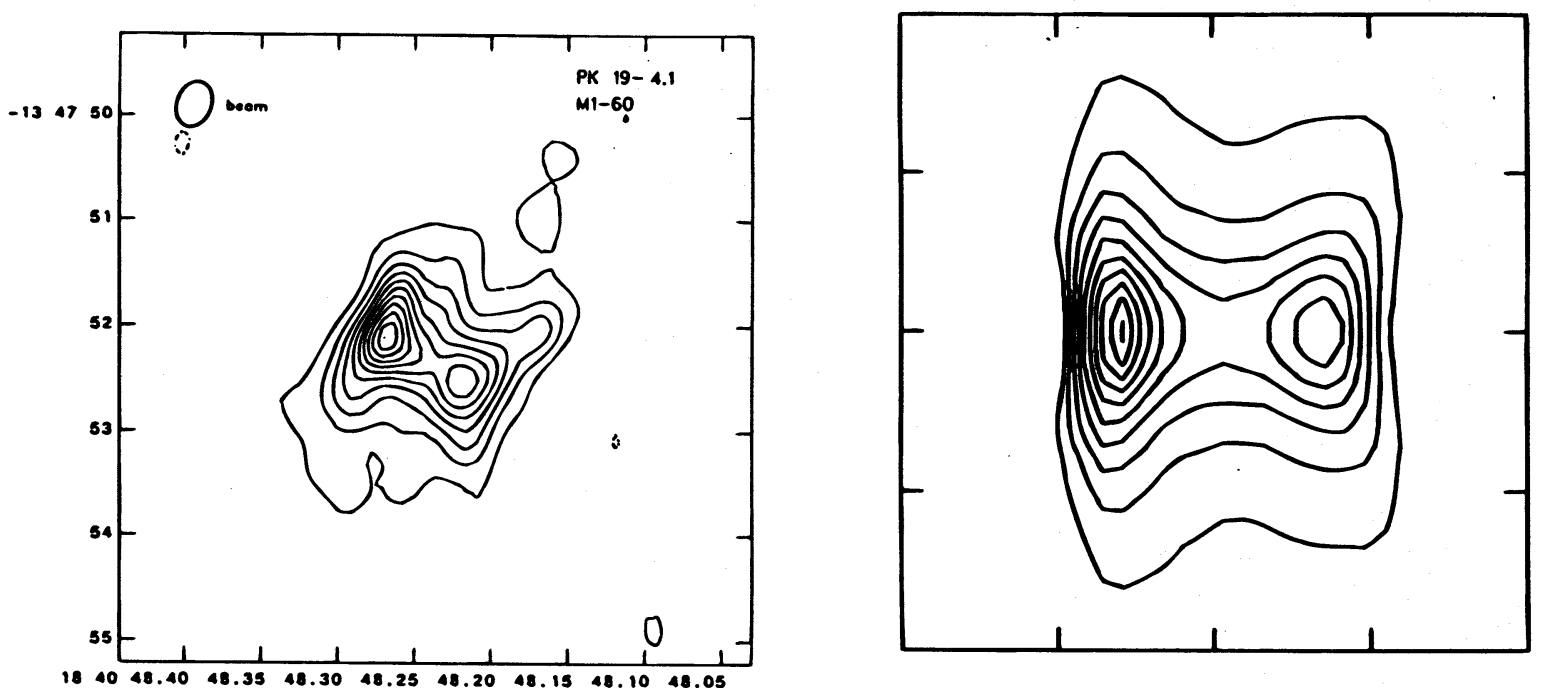

Fig. 4. - Comparison between the model and observed radio images of six compact PNs 


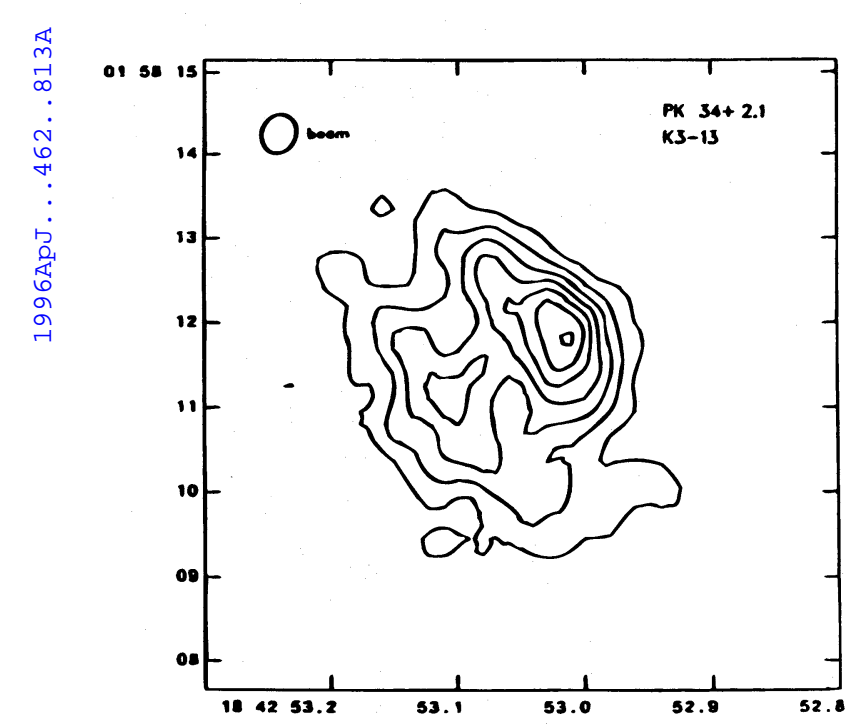

K3-13

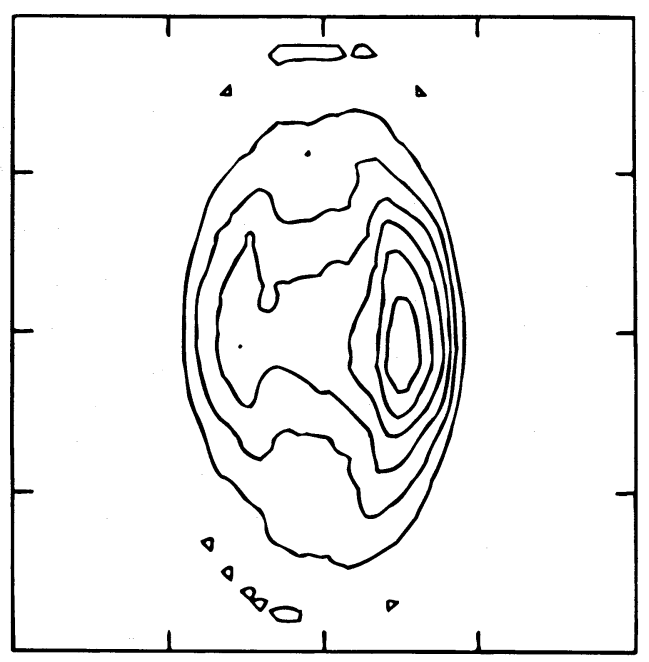

Pc23
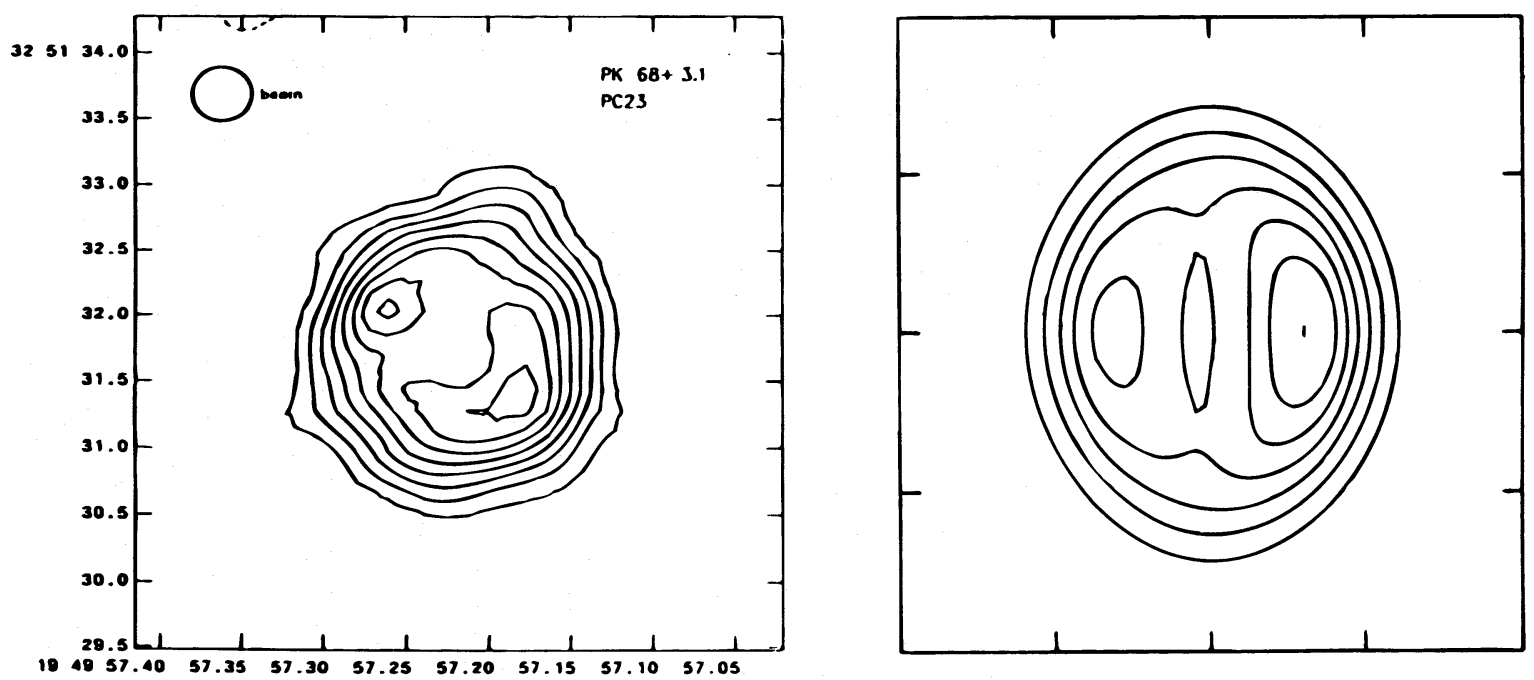

M1-25
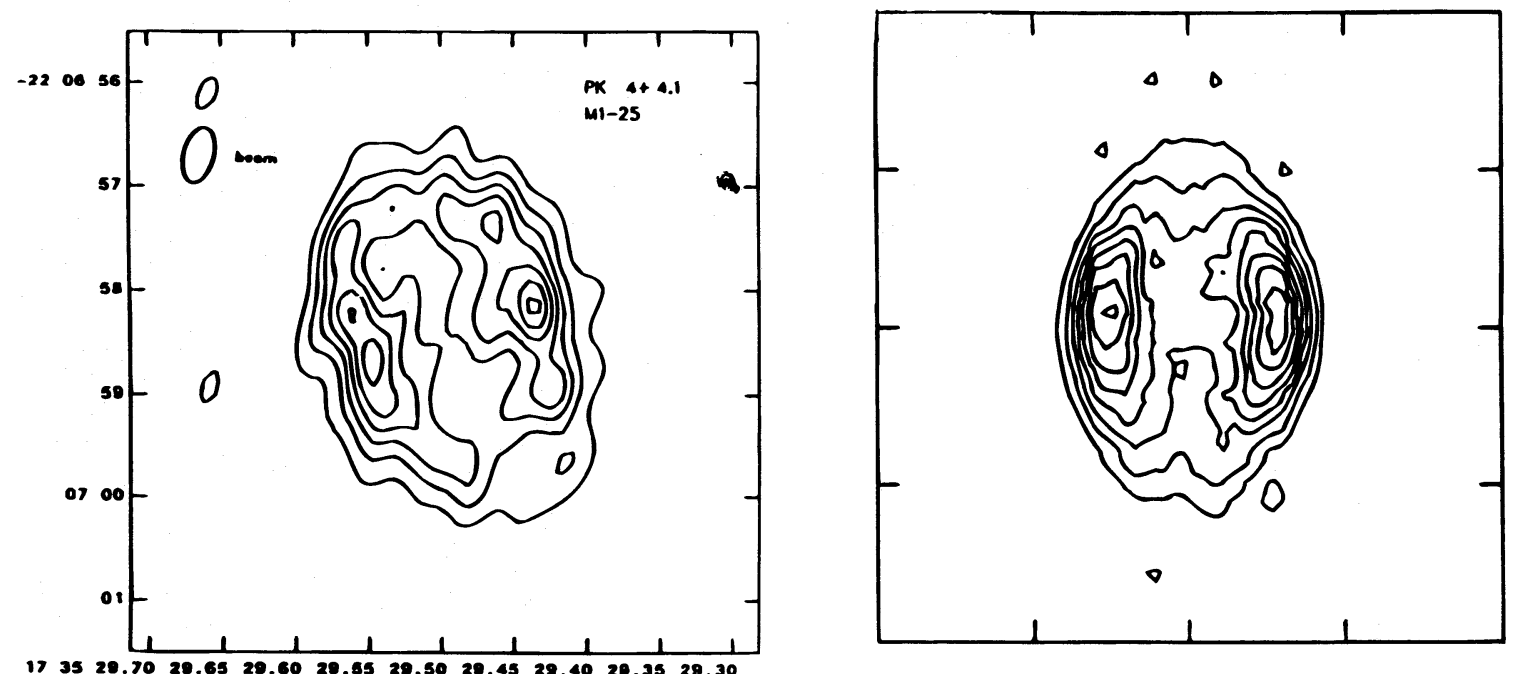

FIG. 4 -Continued 
particular line of sight, defined by the angle $i$ measured from the major axis $\left(i=90^{\circ}\right.$ corresponding to the major axis being perpendicular to the line of sight). For simplicity, we will assume that the nebula is optically thin everywhere, although this is probably not the case for some of the objects. Finally, the resultant two-dimensional image was convolved with a boxcar function, and a contour plot was generated using a standard graphics package.

The apparent shapes of the resulting images are greatly affected by $i, \alpha$, and $\beta$. Figure 3 shows 12 model images for various values of these parameters. For each image, the convolving function was a boxcar of width $2 \delta R_{i}$, and the relative shell thickness $(\Delta)$ and axis ratio $(\epsilon)$ were 0.25 and 2.0, respectively. The model parameters which are represented in the plots are $i=30^{\circ}, 60^{\circ}$, and $90^{\circ}, \alpha=1$ and 4 , and $\beta=0.5$ and 0.1 .

When $\beta$ is not too small, the shell stays thin (relative to $R_{i}$ ) over the entire nebula. If the value of $\beta$ is decreased, the shell widens near the ends of the major axis and the nebula becomes more square in appearance. If the value of $\alpha$ is increased also, then the density drops very quickly as $\theta$ approaches $0^{\circ}$, and the shell widens out closer to the minor axis; this gives the model a distinct butterfly (or an $\mathrm{H}$ or a figure " 8 ") shape. So the appearance of the outer boundary is influenced by $\eta_{\theta}$; this is especially true when the major axis is perpendicular to the line of sight $\left(i \sim 90^{\circ}\right)$. Also, when $i \sim 90^{\circ}$, the inner contours near the minor axis form a characteristic hourglass shape; at $i \sim 60^{\circ}$, the contours begin to flatten and run parallel to the major axis; at $i \leq 30^{\circ}$, the inner and outer contours follow a characteristic " $O$ " or " 0 " appearance, which is not so sensitive to $\eta_{\theta}$. Hence, in principle it is possible to obtain spatial orientation and density information from examining the radio images. However, the larger the convolving beam relative to the size of the nebula, the more difficult it is to discern the density structure and the orientation.

The model was used to try to reproduce some of the well-resolved, high signal-to-noise ratio images in AK90. In order to do this, two additional parameters were introduced to produce a more realistic image. First, all images of PNs in AK90 show varying degrees of random variations from smooth model images. This often makes visual comparisons difficult. These variations are attributable to noise for the weaker objects; for the stronger images, they may be caused by irregularities in the distribution of the nebular material arising from thermal or Rayleigh instabilities. Adding a random value to the flux contribution of each pixel within the ionized structure effectively reproduces this effect. A random number generator with a normal random deviate and zero mean was used to add the "noise"; the variance $(\sigma)$ was adjusted to suit the "noise" level seen in the object. Second, many of the resolved objects show systematic deviation from biaxial asymmetry which cannot be accounted for by a random factor. The deviations range from being very slight, such as in K3-2, He 2-430, and K4-30, to being very severe, such as in IC 2003, J900, and K3-13. These could be caused by density gradients across the nebula, arising from relative motion of the AGB envelope through the interstellar medium, for example. The deviations can be reproduced if $\eta_{\phi}$ and/or $\eta_{\theta}$ are given a slight nonelliptical component. The functions used for this purpose were

$$
\begin{array}{ll}
\eta_{\phi}=\delta_{\phi}+\left(1-\delta_{\phi}\right) \exp [\cos (\phi)-1] & 0 \leq \delta_{\phi} \leq 1, \\
\eta_{\theta}=\delta_{\theta}+\left(1-\delta_{\theta}\right) \exp [\cos (\theta)-1] & 0 \leq \delta_{\theta} \leq 1,
\end{array}
$$

where the $\delta$ values are used to control the degree of ellipsoidal asymmetry along the two axes.

For each model fit, the latitude density indices $(\alpha$ and $\beta$ ), the inclination angle $(i)$, the axis ratio $(\epsilon)$, the relative shell thickness of the minor axis $(\Delta)$, the "noise" level $(\alpha)$, and the asymmetry factor $\left(\delta_{\phi}\right.$ or $\left.\delta_{\theta}\right)$ were adjusted through iteration and visual inspection until a satisfactory representation was achieved. A comparison between observed and simulated images for six nebulae is shown in Figure 4.

Most of the well-resolved images in AK90 can be reproduced quite well using the above approach. Some exceptions are Hu 2-1, which has three emission peaks across the minor axis (Kwok \& Aaquist 1993); J320, which resembles two adjacent emission peaks with a surrounding halo swept toward the northwest; K4-45, which looks like a faint diffuse $\mathrm{H}$ II region; and K3-35, which has a unique S-shape (Aaquist 1993). There are only a few indications of halo structures, so the need to include this in the model is not great. The shell thickness across the minor axis is unresolved for most of the "good" images, so no attempt was made to measure this value very precisely. Also, because of the low surface brightness often found at the ends of the-major axis, the model parameters associated with this axis are often ill defined. For these reasons, no attempt was made to optimize the fits, or to determine the fitting errors in each of the model parameters.

\section{REFERENCES}

Aaquist, O. B. 1993, A\&A, 267, 260

Aaquist, O. B., \& Kwok, S. 1990, A\&AS, 84, 229 (AK90)

. 1991, ApJ, 378, 599

Balick, B. 1987, AJ, 94, 671

Chu, Y., Jacoby, G. H., \& Arendt, R. 1987, ApJS, 64, 529

Curtis, H. D. 1918, Publ. Lick Obs., 13, 57

Evans, D. S., \& Thackeray, A. D. 1950, MNRAS, 10, 37

Frank, A., \& Mellema, G. 1994, ApJ, 430, 800

Frank, A., et al. 1993, ApJ, 404, L25

Greig, W. E. 1971, A\&A, 10, 161

Icke, V. 1988, A\&A, 202, 177

Kahn, F., \& West, K. A. 1985, MNRAS, 212, 837

Khromov, G. S., \& Kohoutek, L. 1968, in IAU Symp. 34, Planetary

Nebulae, ed. D. E. Osterbrock \& C. R. O'Dell (Dordrecht: Reidel), 227

Kwok, S. 1983, AJ, 90, 49

Kwok, S., \& Aaquist, O. B. 1993, PASP, 105, 1456

Kwok, S., Purton, C. R., \& FitzGerald, M. P. 1978, ApJ, 219, L125
Livio, M. 1995, in Asymmetric Nebulae, ed. A. Harpaz \& N. Soker (Ann. Israel Phys. Soc., Vol. 11), 51

Masson, C. R. 1989, ApJ, 346, 243

.1990, ApJ, 348, 580

Mellema, G. 1995, MNRAS, 277, 173

Pottasch, S. R. 1992, in IAU Symp. 131, Planetary Nebulae, ed. S. TorresPeimbert (Dordrecht: Kluwer), 481

. 1995, in Asymmetric Planetary Nebulae, ed. A. Harpaz \& N. Soker (Ann. Israel Phys. Soc., Vol. 11), 7

Schmidt-Voigt, M., \& Köppen, J. 1987a, A\&A, 174, 211

. 1987, A\&A, 174, 223

Schwarz, H. E., Corradi, R. L. M., \& Melnick, J. 1992, A\&AS, 96, 23 (SCM)

Stanghellini, L., Corradi, R. L. M., \& Schwarz, H. E. 1993, A\&AS, 279, 521

Volk, K., \& Kwok, S. 1985, A\&A, 153, 79

Westerlund, B., \& Henize, K. G. 1967, ApJS, 14, 154

Zhang, C. Y., \& Kwok, S. 1993, ApJS, 88, 137

Zijlstra, A. A., Pottasch, S. R., \& Bignell, R. C. 1989, A\&AS, 79, 329 (ZPB) 